\title{
The who, what, and why of research participants' intentions to request a broad range of secondary findings in a diagnostic genomic sequencing study
}

\author{
Christine Rini, $\mathrm{PhD}^{1}$, Cynthia M. Khan, $\mathrm{PhD}^{2}$, Elizabeth Moore, MPH${ }^{3}$, Myra I. Roche, MS, CGC $C^{4,5,6}$, \\ James P. Evans, MD, PhD ${ }^{4,6,7}$, Jonathan S. Berg, MD, PhD ${ }^{4,6}$, Bradford C. Powell, MD, PhD ${ }^{6}$, \\ Giselle Corbie-Smith, MD, MSc ${ }^{7,8,9}$, Ann Katherine M. Foreman, MS, CGC ${ }^{4,6}$, Ida Griesemer, BA ${ }^{10}$, \\ Kristy Lee, MS, CGC 6 , Julianne M. O'Daniel, MS, CGC ${ }^{4,6}$ and Gail E. Henderson, PhD ${ }^{4,8}$
}

\begin{abstract}
Purpose: In a diagnostic exome sequencing study (the North Carolina Clinical Genomic Evaluation by Next-Generation Exome Sequencing project, NCGENES), we investigated adult patients' intentions to request six categories of secondary findings (SFs) with low or no medical actionability and correlates of their intentions.

Methods: At enrollment, eligible participants $(n=152)$ completed measures assessing their sociodemographic, clinical, and literacy-related characteristics. Prior to and during an in-person diagnostic result disclosure visit, they received education about categories of SFs they could request. Immediately after receiving education at the visit, participants completed measures of intention to learn SFs, interest in each category, and anticipated regret for learning and not learning each category.
\end{abstract}

Results: Seventy-eight percent of participants intended to learn at least some SFs. Logistic regressions examined their intention to

\section{INTRODUCTION}

Increasing use of genome and exome sequencing has prompted debate about disclosing secondary findings (SFs) -that is, information unrelated to the indication for sequencing but potentially relevant to individuals' health. ${ }^{1}$ Published recommendations ${ }^{2-4}$ reflect a general consensus that medically actionable SFs (i.e., variants that reach consensus-determined thresholds of clinical validity and clinical utility) should be routinely offered. However, current recommendations do not adequately address disclosure of SFs that have clinical validity but lower medical actionability. These SFs may be valued by people for their personal utility, offering information they judge to be useful in the future. ${ }^{5}$ Understanding people's preferences for learning SFs with lower medical actionability, and factors associated with these preferences, will inform recommendations, policy, and development of educational and counseling approaches to support informed decision making. learn any or all of these findings (versus none) and interest in each of the six individual categories (yes/no). Results revealed little association between intentions and sociodemographic, clinical, or literacy-related factors. Across outcomes, participants who anticipated regret for learning SFs reported weaker intention to learn them (odds ratios (ORs) from 0.47 to 0.71 ), and participants who anticipated regret for not learning these findings reported stronger intention to learn them (OR 1.61-2.22).

Conclusion: Intentions to request SFs with low or no medical actionability may be strongly influenced by participants' desire to avoid regret.

Genet Med advance online publication 26 October 2017

Key Words: anticipated regret; behavioral intentions; diagnostic genomic sequencing; incidental findings

Evidence across various populations suggests that most people initially express interest in all possible SFs-even those with lower medical actionability. This response is observed both when patients are offered this information ${ }^{6-10}$ and when they are considering a hypothetical question of whether they would want it if it were available. ${ }^{11-13}$ People often demonstrate different levels of interest in different types of $\mathrm{SFs},{ }^{6-8,12-14}$ suggesting that some features of $\mathrm{SFs}$ are distinguished at least by some people.

Researchers have sought to identify correlates of preferences for learning SFs of various types. Sociodemographic characteristics often have null or weak associations with preferences for learning $\mathrm{SFs},{ }^{10,15,16}$ as do individual-level clinical characteristics such as prior genetic testing, role as a patient or patient's guardian, or patient diagnoses. ${ }^{8,10,15}$ Other research has examined psychological correlates. One study of advanced cancer patients' preferences for learning SFs from somatic and germ-line sequencing ${ }^{10}$ found that patients with more positive attitudes about genetic testing had a higher

\footnotetext{
${ }^{1}$ John Theuer Cancer Center, Hackensack University Medical Center, Hackensack, New Jersey, USA; ${ }^{2}$ Econometrica, Inc., Bethesda, Maryland, USA; ${ }^{3}$ Blue Cross and Blue Shield of North Carolina, Durham, North Carolina, USA; ${ }^{4}$ Center for Genomics and Society, University of North Carolina, Chapel Hill, North Carolina, USA; ${ }^{5}$ Department of Pediatrics, University of North Carolina, Chapel Hill, North Carolina, USA; ${ }^{6}$ Department of Genetics, University of North Carolina at Chapel Hill, Chapel Hill, North Carolina, USA; ${ }^{7}$ Department of Medicine, University of North Carolina at Chapel Hill, Chapel Hill, North Carolina, USA; ${ }^{8}$ Department of Social Medicine, University of North Carolina at Chapel Hill, Chapel Hill, North Carolina, USA; ${ }^{9}$ Center for Health Equity Research, University of North Carolina at Chapel Hill, Chapel Hill, North Carolina, USA; ${ }^{10}$ Department of Health Behavior, University of North Carolina at Chapel Hill, Chapel Hill, North Carolina, USA. Correspondence: Christine Rini (christine.rini@hackensackmeridian.org) 
preference for learning both somatic and germ-line findings. This study investigated SFs with clear medical actionability, but also a very limited domain of SFs with lower medical actionability in a population with unique concerns because of their advanced disease. It is unclear whether these findings will generalize to broader patient populations or to a broader set of SFs with lower medical actionability.

Other relevant evidence comes from a large-scale pilot study investigating use of genomic sequencing to identify genetic risk for heart disease. ${ }^{17}$ Participants were older adult community resident volunteers; nearly all were white and well-educated and had a high income. The investigators examined participants' intentions to learn medically actionable SFs and other SFs including carrier status and gene variants with uncertain meaning for health. Generally speaking, participants with positive attitudes toward SFs and stronger perceptions that others thought they should learn them had stronger intentions to learn SFs. ${ }^{7}$ Participants with higher dispositional optimism and greater perceived risk for common diseases also had stronger intentions to learn SFs. ${ }^{18}$ Conversely, participants who believed they were more likely to be emotionally devastated or unable to cope if they learned threatening information ${ }^{15}$ or perceived the findings to be more ambiguous (i.e., relatively low in interpretability, trustworthiness, and accuracy) ${ }^{19}$ had a weaker intention to learn SFs. Effects of perceived ambiguity were modified by dispositional factors (e.g., medical ambiguity aversion). These findings reveal associations between psychological factors and intentions to learn SFs, but apply to a limited domain of SFs with lower medical actionability. The study's low racial/ethnic and socioeconomic diversity also raises questions about generalization to more diverse samples.

\section{Overview of the present study}

Data for the present study were from the North Carolina Clinical Genomic Evaluation by Next-Generation Exome Sequencing (NCGENES) project, which is investigating integration of diagnostic exome sequencing and return of SFs into clinical practice. ${ }^{20}$ Participants were child and adult patients enrolled to undergo diagnostic exome sequencing to determine the etiology of a suspected genetic disorder. Additionally, intellectually competent adult patient participants who did not receive medically actionable SFs were entered into a randomized study of decision making regarding six categories of SFs with lower medical actionability. The present study investigated intentions to request these SFs in participants randomized to have the option to request them. Intentions reliably predict substantial variance in subsequent behavior, ${ }^{21}$ revealing an inclination to make a particular decision. Thus, they provide a theory-based foundation for understanding and supporting informed decision making. For instance, antecedents of intentions such as patients' attitudes about learning SFs (i.e., beliefs about the value and consequences of learning them), perceived social norms concerning this decision (i.e., beliefs about others' approval of their decision, or others' decisions), and self-efficacy (i.e., confidence in their ability to make a good decision or handle the consequences of their decision) ${ }^{22}$ could be incorporated into a shared decision-making process.

We also investigated factors that may influence participants' intentions, focusing on factors that could be pragmatically evaluated in clinical settings: sociodemographic factors, individual-level clinical factors, literacy-related factors that may affect patients' understanding of and comfort with making decisions about complex genetic information, and anticipated regret for learning and not learning the secondary information. Anticipated regret and similar emotionallyfocused factors are increasingly incorporated into health decision-making research to enrich evidence that previously focused on cognition and rational decision processes. ${ }^{23}$ Evidence suggests that people are often highly motivated to reduce uncertainty and to avoid feared and/or unpleasant outcomes. ${ }^{23}$ The aim of this investigation was to guide recommendations and strategies for offering and disclosing results from a potentially vast and heterogeneous set of SFs with lower medical actionability.

\section{Participants}

\section{MATERIALS AND METHODS}

Adult and pediatric patients with health conditions suspicious for a monogenic etiology were referred by clinicians at the University of North Carolina Hospitals (Chapel Hill, NC), Moses Cone Memorial Hospital (Greensboro, NC), and Vidant Medical Systems (Greenville, NC). All had exome sequencing and received diagnostic results and, if identified, medically actionable SFs. ${ }^{3}$ Adult patient participants who were able to provide informed consent and who did not have a medically actionable SF were randomized into either a group that had the option to request SFs with lower medical actionability (decision arm) or one that did not have this option (control arm). The present study included only those randomized to the decision group ( $n=152$ as of January 2016). Thus, participants were cognitively intact adults who had not received medically actionable SFs and who were randomized to have the option to learn SFs with lower medical actionability.

\section{Categories of secondary findings}

SFs with lower medical actionability were grouped into six categories based on the expert consensus of genetics professionals on the study team: ${ }^{24}$ (i) single-nucleotide polymorphisms associated with change in risk for a variety of multifactorial diseases, (ii) pharmacogenomic variants, (iii) variants that identified carrier status for recessive disorders, (iv) $A P O E$ variants associated with risk for Alzheimer disease, (v) variants associated with Mendelian diseases for which no effective presymptomatic intervention exists, and (vi) variants associated with Mendelian diseases that manifest with a severe, progressive neurodegenerative course and that cannot be prevented or effectively treated. These categories were used for in-person and printed patient education (see Table 1). 
Table 1 Categories of secondary findings and summary of information provided in NCGENES patient-education brochure

\begin{tabular}{|c|c|c|c|}
\hline Category & Description from brochure & Examples provided & $\begin{array}{l}\text { How patients learn } \\
\text { findings after call to } \\
\text { request them }\end{array}$ \\
\hline $\begin{array}{l}\text { A. Single-nucleotide polymorphisms (SNPs) } \\
\text { associated with increased risk for a variety } \\
\text { of common diseases }\end{array}$ & $\begin{array}{l}\text { "Variants, called SNPs (pronounced } \\
\text { "snips"), that may slightly affect your } \\
\text { chance for developing common conditions } \\
\text { like heart disease, cancer, and diabetes." }\end{array}$ & $\begin{array}{l}\text { - Heart disease } \\
\text { - Cancer } \\
\text { - Diabetes }\end{array}$ & - Telephone \\
\hline C. Carrier status for recessive conditions & $\begin{array}{l}\text { "Variants in genes that do not usually affect } \\
\text { your health but that increase the risk for } \\
\text { health problems in your children and others } \\
\text { in future generations." }\end{array}$ & $\begin{array}{l}\text { - Cystic fibrosis } \\
\text { - Sickle cell anemia }\end{array}$ & - One in-person visit \\
\hline $\begin{array}{l}\text { E. Risk for rare Mendelian diseases for } \\
\text { which no effective presymptomatic } \\
\text { intervention exists }\end{array}$ & $\begin{array}{l}\text { "Rare variants in genes that directly cause } \\
\text { you to have an increased risk for a genetic } \\
\text { disease that cannot be prevented, but that } \\
\text { may have some treatments after symptoms } \\
\text { develop." }\end{array}$ & $\begin{array}{l}\text { - Thrombophilia } \\
\text { - Polycystic kidney } \\
\text { disease }\end{array}$ & - One in-person visit \\
\hline $\begin{array}{l}\text { F. Risk for very rare, highly penetrant } \\
\text { Mendelian diseases that cannot be } \\
\text { prevented or effectively treated and } \\
\text { manifest with a progressive } \\
\text { neurodegenerative course }\end{array}$ & $\begin{array}{l}\text { "Very rare variants in genes that directly } \\
\text { cause severe and progressive diseases of the } \\
\text { brain and nervous system that cannot be } \\
\text { prevented and that have no effective } \\
\text { treatments after symptoms develop." }\end{array}$ & $\begin{array}{l}\text { - Amyotrophic lateral } \\
\text { sclerosis }\end{array}$ & - Two in-person visits \\
\hline
\end{tabular}

NCGENES, North Carolina Clinical Genomic Evaluation by Next-Generation Exome Sequencing project.

\section{Procedures}

Staff recruited patients to NCGENES by phone and scheduled an enrollment visit. These potential participants completed a mailed intake questionnaire and brought it to their enrollment visit, at which they met with a board-certified genetic counselor to provide informed consent and blood samples for sequencing. Randomization to the decision or control group occurred after participants' diagnostic results were ready, 612 months later. Participants were informed of their group assignment in a letter confirming their appointment for an inperson return of results visit. For decision arm participants, this mailing included a brochure describing "non-medically actionable" SFs, the six categories and examples of conditions in each, and potential benefits and harms of learning SFs from each category. These descriptions were designed by clinicians and researchers on the study team to present a balanced discussion of pros and cons, and the brochure included a values clarification exercise to promote informed decision making; participants were invited to record their reasons for learning and for not learning each category, then summarize these reasons in a chart they could bring to their return of results visit. At this visit, participants first learned their diagnostic results in a meeting with a geneticist, usually accompanied by a certified genetic counselor. The clinicians then shifted to educating them about SFs, reiterating and expanding on information in the brochure. Participants were informed that they had to call the study coordinator to request SFs, initiating analysis of the requested categories (i.e., their SFs had not already been analyzed), and that when their results were ready, staff would call them to schedule an appointment to disclose requested SFs by phone or in person, depending on categories requested. Prior to leaving the clinic appointment, participants completed a post-return of results interview and questionnaire. All procedures were approved by the institutional review boards of the University of North Carolina, Chapel Hill, and the Vidant Medical System.

\section{Measures}

Sociodemographic variables (patient sex, age, race, ethnicity, educational attainment, annual household income, marital status, and health insurance status) were obtained from health records or were self-reported in the intake questionnaire.

Clinical variables other than type of diagnostic result were measured in the intake questionnaire. Physical functioning was assessed with the self-report Karnofsky Performance Status scale, ${ }^{25,26}$ which includes eight categories from "I am 
severely disabled and I require hospitalization and continuous nursing care" to "I am able to carry on normal activity or do work, and I have no physical complaints or problems" (coded $1-8$, with higher scores indicating better functioning). Prior genetic testing was assessed with the question: "Have you ever had a genetic test? (it's usually a blood test)" (yes/no). Types of diagnostic result were positive (findings revealed an explanation for the health concern), negative (findings did not reveal an explanation for the health concern), or uncertain (findings could possibly explain the health concern). A dichotomous variable for each type indicated whether it had been found for each participant (yes/no).

\section{Literacy variables}

General health literacy was assessed in person at enrollment using the 66-item Rapid Estimate of Adult Literacy Measure. ${ }^{27}$ A literacy score was created by summing the number of words pronounced correctly. Participants were categorized as low literacy (score of $0-60$; less than ninthgrade reading level) or high literacy (score of 61-66; ninthgrade reading level or higher), ${ }^{28}$ making use of recommended cutoffs but collapsing across categories indicating low literacy because of the small proportion of these patients. Objective numeracy was assessed in the intake questionnaire with a validated measure that presented three math problems involving proportions, fractions, and percentages. ${ }^{29}$ Correct responses were summed to create a numeracy score. Secondary findings knowledge was assessed in the postreturn of results questionnaire with 12 items created for this study assessing knowledge of facts covered in our brochure and in-person counseling (e.g., "Learning this type of information will usually tell you for certain whether or not you will get a health problem in the future"). Participants marked each item "true," "false," or "not sure/don't know." We summed correct responses to create a knowledge score, with "not sure/don't know" responses considered incorrect (Cronbach's alpha $=0.69$ ).

Anticipated regret was assessed in the post-return of results interview with a measure ${ }^{30}$ adapted to evaluate anticipated regret for not learning and for learning each of the six categories of SFs (12 items total). The interviewer described a category and asked: "Imagine that you got a health problem that you believe this information could have prepared you for or helped you avoid. How much would you regret not choosing to learn this information?" or "Imagine that you chose to get this information and you found out something about your health you did not want to know. How much would you regret choosing to learn this information?" The order of these questions was randomized across participants. Responses ranged from $1=$ not at all to $5=$ very much and were averaged to create scores indicating anticipated regret for not learning SFs and for learning SFs (alphas $=0.91$ and 0.90, respectively).

Intention to learn SFs was assessed in the post-return of results interview. Participants were instructed to consider the categories of SFs together and to report how likely it was that they would decide to learn any of them using the following scale: $1=$ you definitely will not, $2=$ you probably will not, $3=$ you're not sure, $4=$ you probably will, and $5=$ you definitely will. Participants answering 4 or 5 were asked which categories they would be interested in learning (scored $1=$ interested and $0=$ not interested for each category). Interviewers emphasized that their decision was not final.

\section{Analyses}

We conducted descriptive analyses and evaluated the psychometric properties of all measures. Missing income data were replaced using the median income for North Carolina from 2014 (which approximated our sample's median income). ${ }^{31}$ Missing dichotomous general health literacy data were replaced using participants' educational attainment; those with less than a ninth-grade education were entered as having low literacy. Remaining missing data were minimal and were replaced with mean and mode imputation, as appropriate. The dichotomous primary outcome was intention to learn some or all SFs versus no SFs. Interest in learning each of the six categories of SFs were secondary outcomes. We determined which variables to enter into logistic regression analyses predicting these outcomes by examining correlations between the outcomes and sociodemographic, clinical, literacy, and anticipated regret variables. These variables were entered into a model for a particular outcome if they were correlated with the outcome at $P<0.10$.

\section{RESULTS}

Descriptive statistics are shown in Table 2. Correlations among study variables are shown in Table 3.

\section{Participant characteristics}

About three-quarters of participants were women and nearly one-quarter identified as Hispanic and/or nonwhite. Average age was 47 years. Fewer than half had a 4 -year college degree or graduate education. Participants' mean income fell between $\$ 45,000$ and $\$ 59,999$. Their clinical characteristics and literacy varied considerably.

Seventy-six percent of participants intended to learn some or all categories of SFs, including $38 \%$ who intended to learn all six categories and $38 \%$ who intended to learn some but not all categories. More than half were interested in learning category A (61\%), B (63\%), C (61\%), D (61\%), and E (55\%). Slightly fewer were interested in category F (49\%). For participants who intended to learn some but not all categories, combinations of categories they were interested in learning were highly heterogeneous, with no predominant pattern.

Participants' average anticipated regret scores corresponded to anticipating that they would regret learning these findings between "a little bit" and "somewhat" and anticipating that they would regret not learning these findings between "somewhat" and "quite a bit." The moderate negative correlation between these scores suggested that it is possible to anticipate regret from both learning and not learning these findings. A paired-samples t-test indicated that participants 
Table 2 Participant characteristics $(N=152)$

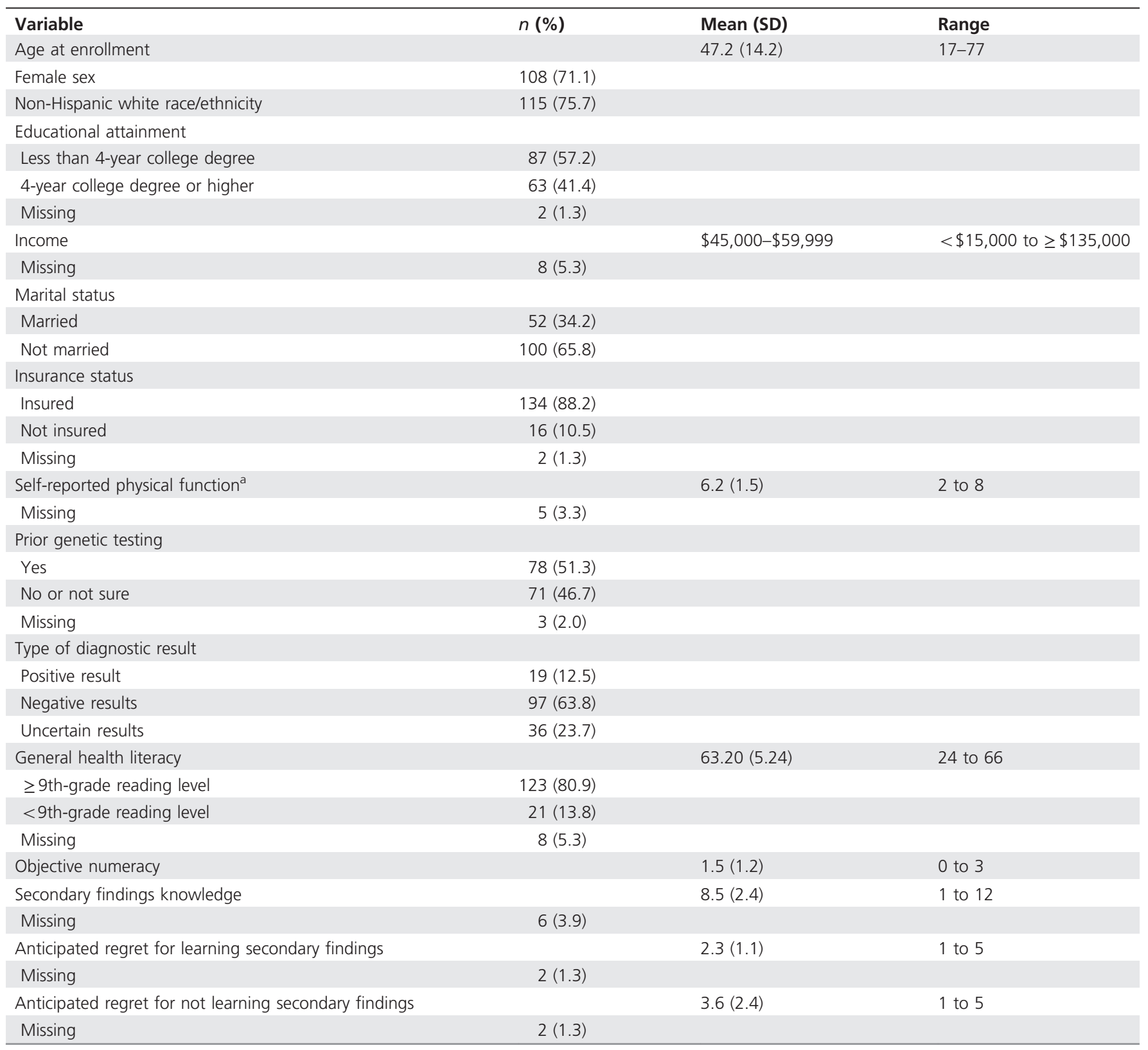

${ }^{a}$ On a scale from 1 to 8 , with 8 indicating the best physical functioning.

anticipated greater regret for not learning SFs than for learning them $(P<0.001)$.

\section{Intention to learn secondary findings from some or all (versus no) categories}

When potential predictors (Table 3) were entered into a logistic regression to evaluate their independent effects controlling for other variables in the model (Table 4), findings indicated that having higher general health literacy, higher numeracy, and higher anticipated regret for not learning SFs were associated with greater likelihood of intending to learn some or all SFs. Anticipated regret for learning SFs was associated with lower likelihood of intending to learn SFs. Secondary findings knowledge was not associated with this outcome.

Interest in learning secondary findings from each category These logistic regression analyses are described below and summarized in Table 5. Across all categories of SFs, anticipated regret for not learning the category was associated with greater interest in learning these findings (all $P<0.001$ ) and anticipated regret for learning the category was independently associated with lower interest in learning them ( $P$ from 0.036 to $<0.001$ ). With a few 


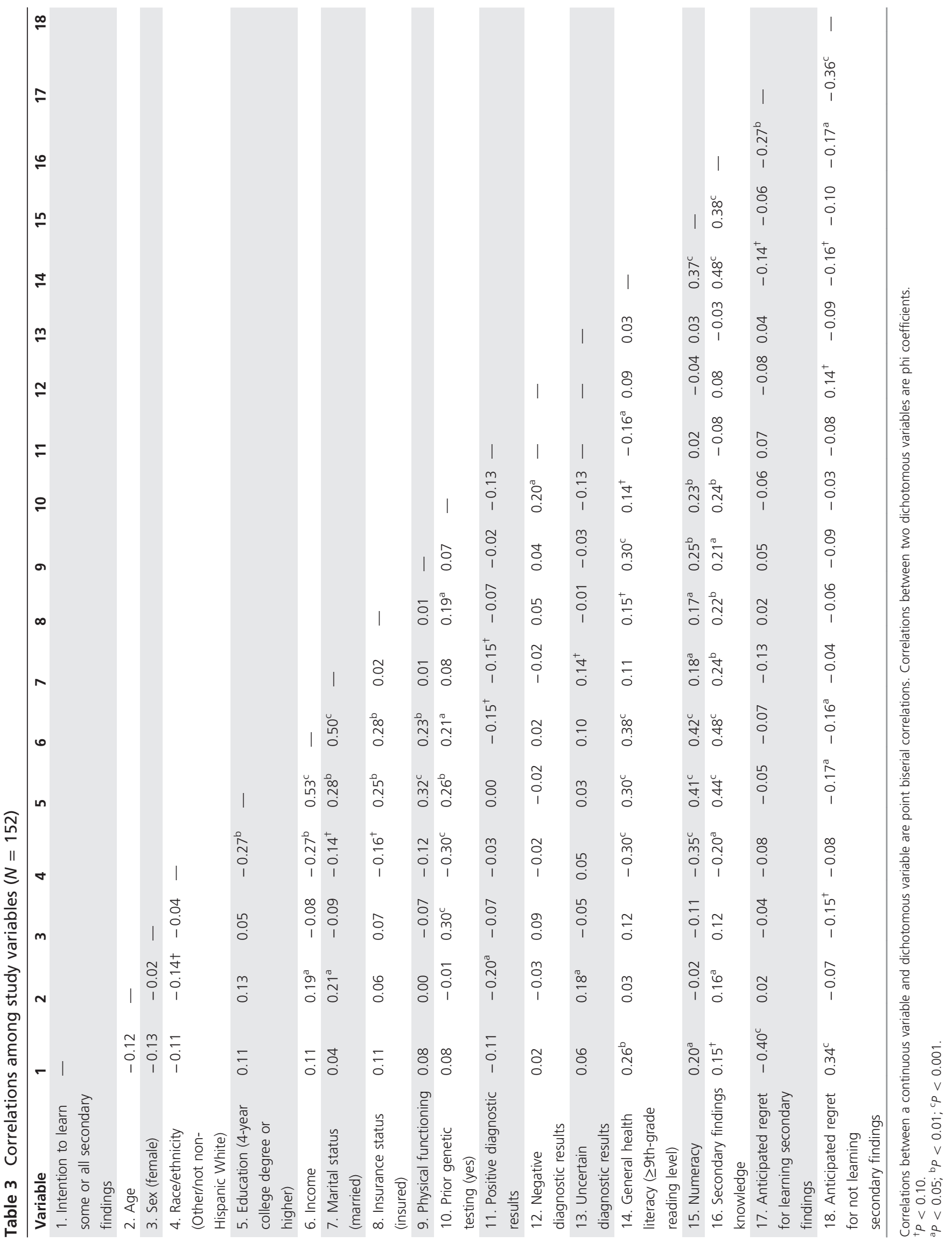


Table 4 Logistic regression analysis predicting intention to learn some or all secondary findings with low or no medical actionability (versus none) $(N=152)$

\begin{tabular}{|c|c|c|c|c|c|}
\hline Variable & B & SE & OR & $95 \% \mathrm{Cl}$ & $P$ \\
\hline High general health literacy ( $\geq 9$ th-grade reading level) & 1.89 & 0.68 & 6.61 & $1.76,24.85$ & 0.005 \\
\hline Numeracy & 0.46 & 0.23 & 1.59 & $1.01,2.49$ & 0.044 \\
\hline Secondary findings knowledge & -0.09 & 0.12 & 0.91 & $0.72,1.15$ & 0.431 \\
\hline Anticipated regret for learning secondary findings & -0.75 & 0.23 & 0.47 & $0.3,0.74$ & 0.001 \\
\hline Anticipated regret for not learning secondary findings & 0.8 & 0.22 & 2.22 & $1.44,3.42$ & $<0.001$ \\
\hline
\end{tabular}

$\mathrm{B}$, unstandardized regression weight; $\mathrm{Cl}$, confidence interval; OR, odds ratio; SE, standard error of $\mathrm{B}$.

exceptions, other factors did not help explain participants' interest in learning these categories. For category C, greater numeracy was associated with greater interest in learning the findings $(P=0.026)$. For category $\mathrm{E}$, women were less likely than men to intend to learn these findings $(P=0.01)$. Having had a prior genetic test was associated with greater interest in learning category $\mathrm{E}(P=0.02)$.

\section{DISCUSSION}

In a sample of adult patients undergoing diagnostic exome sequencing, we investigated participants' intentions to learn some or all SFs with lower medical actionability and a broad set of correlates of their intentions. Approximately three out of four participants intended to learn at least some of these SFs. Participants were more likely to report this intention if they had higher general health literacy and greater numeracy, if they more strongly anticipated that they would regret not learning this information, and if they anticipated that they were relatively unlikely to regret learning it. We found similar results for each of the six categories of SFs, with few exceptions. Thus, forming an intention to learn SFs with lower medical actionability was largely motivated by a desire to avoid a future negative emotional state- regret $^{23}$-rather than membership in sociodemographic or clinical subgroups.

Participants anticipated greater regret for not learning than for learning the SFs we studied, consistent with research in other populations ${ }^{32}$ and possibly helping to explain why most participants' initial response was to intend to learn at least some of their SFs. Their interest in learning these findings is consistent with other studies using different populations and research methods. ${ }^{4,6-13}$ The effect size of anticipated regret for not learning SFs ("anticipated inaction regret") was large, similar to large effects for anticipated inaction regret found in a meta-analysis of the broad body of research on healthrelated intentions (e.g., for behaviors such as vaccination, cancer screening, and safe sex practices), ${ }^{32}$ whereas the effect size of anticipated regret for learning SFs ("anticipated action regret") was small compared with effect sizes for anticipated action regret in that same meta-analysis. Thus, effects of anticipating regret for learning SFs with lower medical actionability were weak compared with the larger literature. Despite in-person and printed education describing both potential harms and limited clinical utility, patients appeared to perceive few downsides in learning this information.
Converging evidence shows that some people view SFs as valuable for protecting their own and their family's health, that they perceive SFs as potentially useful in the future, and that they feel that having knowledge is empowering. ${ }^{33,34}$ Thus, our participants' intentions to learn this information was consistent with the idea that they perceived it as having personal utility, despite education regarding its lack of tangible medical actionability and the limits of genomic sequencing and current knowledge of genetic causes of disease. ${ }^{35,36}$ Personal utility may be an important type of attitude affecting behavioral intentions. According to the theory of planned behavior and similar theories, it may therefore play a causal role in determining behavioral intentions and subsequent behavior. ${ }^{22}$

The two forms of anticipated regret we investigated compete with each other: one motivates patients to consider learning their SFs whereas the other motivates the opposite choice. Given their moderate association, some participants anticipated regret for both getting and not getting this information, leaving them ambivalent about this decision. Ambivalence can affect decision making in complex ways. ${ }^{37}$ There may also be practical and other barriers to following through on intentions to learn SFs. Forthcoming analyses of NCGENES participants' decisions will clarify how intentions to request SFs of lower medical actionability translate to actual requests for these SFs. An important goal will be to consider the role of various potential barriers to action and other factors that may influence the link between intentions and behavior. Although behavioral intentions reliably predict a substantial proportion of variance in subsequent behavior, understanding the factors that influence the strength of this association ${ }^{22}$ in the context of SFs will provide useful insight into circumstances under which patients' initial intentions will and will not translate into actual requests.

The relatively small percentage (38\%) of our participants who intended to learn all of their SFs contrasts with larger percentages in other studies. ${ }^{6,13,14}$ One explanation for this discrepancy may be our tiered consent: we educated participants about SFs and asked them to make a decision about learning the six categories only after they had received their diagnostic results, thereby separating the desire for a diagnostic result from a desire for SFs. Participants also had a relatively open-ended time frame to make decisions, eliminating pressure to risk losing their chance. Our approach 


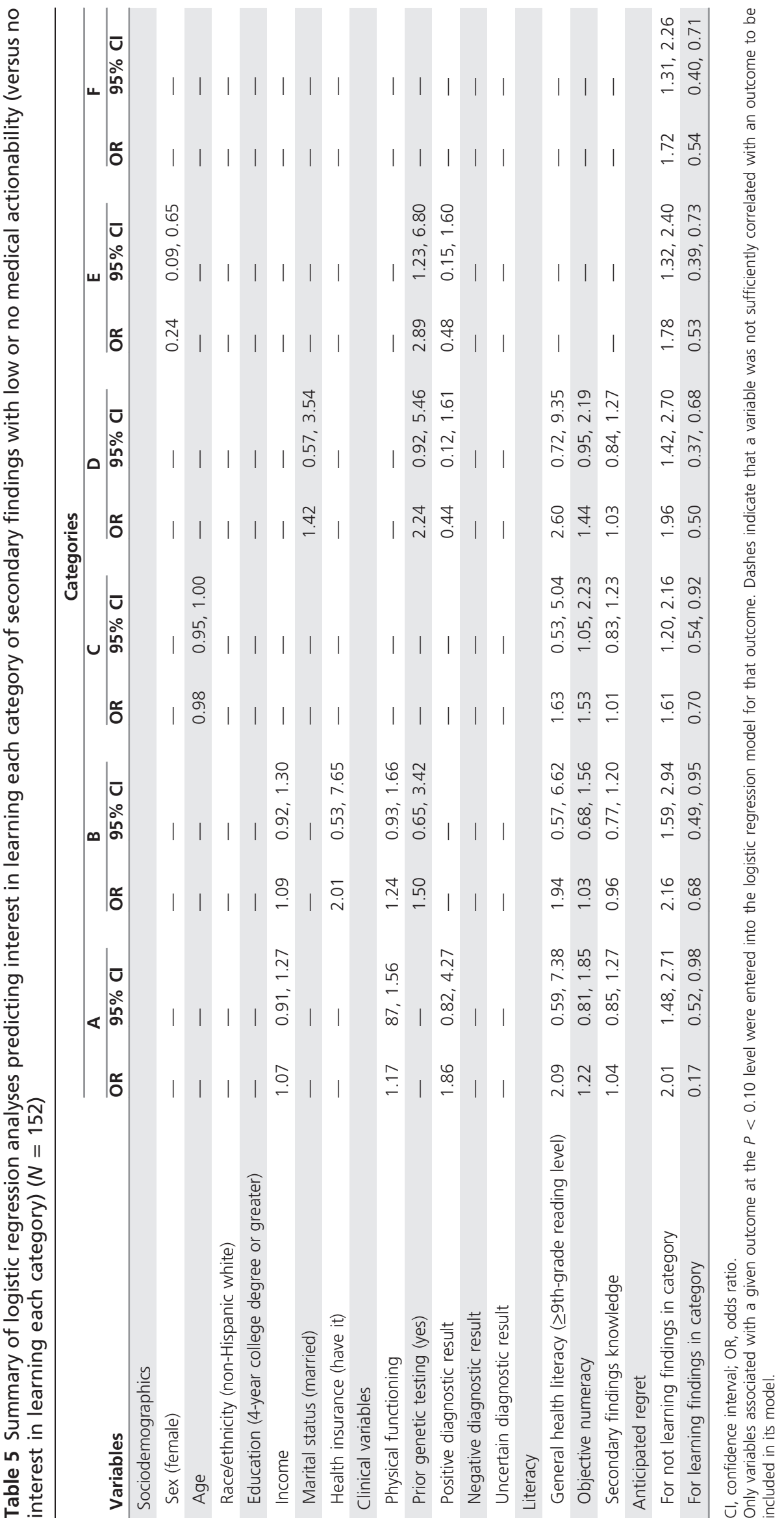


contrasts with more typical current clinical practice in which these decisions are made at the time of consent for sequencing (e.g., due to laboratory consent forms and protocols) limiting both the time patients have to consider their decision and opportunity to provide them with education and counseling about their options. Finally, we clearly communicated that analysis of SFs would only be performed if participants specifically requested them, emphasizing that we were not in possession of knowledge they were not yet privy to. Although it is not clear whether patients understand what a laboratory may or may not know about their SFs before any request is made, these procedures may have increased the likelihood that our participants would weigh the value of available SFs and balance their desire to get them with competing goals (e.g., managing the implications of their diagnostic results).

There were several study limitations. First, we did not evaluate the influence of study clinicians who counseled participants, nor did we standardize or script their presentation of information about SFs. Although the clinicians may have varied somewhat in their presentation, we believe that any such variation did not influence our findings. The clinicians reiterated and expanded upon information in the study brochure, which they helped develop and which offered a balanced discussion of potential harms and limited benefits of each category. Also, most sessions were led by both a geneticist and a certified genetic counselor with formal training in avoiding being directive. However, further research on patient-provider communications and the effects of variation on anticipated regret in clinical settings is warranted, particularly because variation in "real world" settings would be important for designing clinical support. Second, our measure of SF knowledge was developed for this study and thus not validated. Its internal consistency (0.69) fell just under the accepted rule-of-thumb cutoff for good internal consistency (0.70), suggesting the need for research using a refined measure. Third, we investigated participants' intention to request SFs rather than their actual requests. Analyses investigating actual requests and the link between intentions and subsequent behavior are underway-a critical research question in light of evidence that in some areas of genomics intentions do not have a strong association with subsequent behaviors. $^{21}$ Yet, investigation of behavioral intentions is important in its own right, particularly because we assessed intentions at a time that offers an ideal opportunity to intervene to ensure informed decision making prior to actual requests for SFs. Finally, pediatric patients, adult patients with cognitive impairment, and adult patients who received medically actionable SFs were not eligible for randomization. Thus, our findings apply to cognitively intact adult patients. Parents of pediatric or cognitively impaired adult patients may have different perspectives.

The study also had notable strengths. Our approaches to categorizing SFs, educating participants about them, and allowing participants to request them were designed to reflect a clinically realistic, reasonable, and ethical protocol that would provide useful guidance for policy and for development of educational and counseling interventions. Furthermore, our sample included a diverse set of diagnoses and sociodemographic diversity, enhancing the generalizability of our results. We examined intentions in an actual decision, avoiding response biases that may affect hypothetical decisions. We also investigated a broad set of potential SFs, allowing us to evaluate intentions and correlates of those intentions across categories with different clinical and psychosocial risk profiles. Finally, we studied a broader set of potential correlates of participants' intention to learn SFs than has been investigated in the past, focusing on those that could be evaluated by a clinician to help guide patient education and counseling.

In conclusion, our findings suggest avenues for future research and development of patient education and counseling interventions, given that behavioral intentions predict subsequent health behavior ${ }^{38}$ and greater anticipated regret may strengthen the link between intention and behavior. ${ }^{39}$ Genetic counselors are trained to prompt patients to consider and probe for anticipated regret, ${ }^{40}$ and other clinicians could learn this technique. These findings may also guide recommendations for returning SFs in a way that balances patients' desire to learn SFs with practicality, costs, and burden on the health-care system and research budgets. To the extent their desire is prompted by the goal of avoiding negative emotional outcomes, it is worthwhile to consider ways to balance this desire with information that improves their ability to make informed decisions.

\section{ACKNOWLEDGMENTS}

The research on which this study is based was funded by the National Human Genome Research Institute of the National Institutes of Health under award U01 HG006487-01. The content is solely the responsibility of the authors and does not necessarily represent the official views of the National Institutes of Health. Dr. Corbie-Smith's time on this project was supported by grant funding from 2K24 HL105493-06-07 and 5UL1 TR001111-01-03.

\section{DISCLOSURE}

The authors declare no conflict of interest.

\section{REFERENCES}

1. Roche MI, Berg JS. Incidental findings with genomic testing: implications for genetic counseling practice. Curr Genet Med Rep. 2015;3:166-176.

2. Green RC, Berg JS, Grody WW, et al. ACMG recommendations for reporting of incidental findings in clinical exome and genome sequencing. Genet Med. 2013;15:565-574.

3. Berg JS, Khoury MJ, Evans JP. Deploying whole genome sequencing in clinical practice and public health: meeting the challenge one bin at a time. Genet Med. 2011;13:499-504.

4. Berg JS, Amendola LM, Eng C, et al. Processes and preliminary outputs for identification of actionable genes as incidental findings in genomic sequence data in the Clinical Sequencing Exploratory Research Consortium. Genet Med. 2013;15:860-867.

5. Grosse SD, McBride CM, Evans JP, Khoury MJ. Personal utility and genomic information: look before you leap. Genet Med. 2009;11: 575-576.

6. Jelsig AM, Qvist N, Brusgaard K, Ousager LB. Research participants in NGS studies want to know about incidental findings. Eur J Hum Genet. 2015;23:1423-1426. 
7. Facio FM, Eidem $H$, Fisher $\mathrm{T}$, et al. Intentions to receive individual results from whole-genome sequencing among participants in the ClinSeq study. Eur J Hum Genet. 2013;21:261-265.

8. Bishop CL, Strong KA, Dimmock DP. Choices of incidental findings of individuals undergoing genome wide sequencing, a single center's experience. Clin Genet. 2017;91:137-140.

9. Shahmirzadi L, Chao EC, Palmaer E, Parra MC, Tang S, Gonzalez KD. Patient decisions for disclosure of secondary findings among the first 200 individuals undergoing clinical diagnostic exome sequencing. Genet Med. 2014;16:395-399.

10. Gray SW, Park ER, Najita J, et al. Oncologists' and cancer patients' views on whole-exome sequencing and incidental findings: results from the CanSeq study. Genet Med. 2016;18:1011-1019.

11. Hitch K, Joseph G, Guiltinan J, Kianmahd J, Youngblom J, Blanco A. Lynch syndrome patients' views of and preferences for return of results following whole exome sequencing. J Genet Couns. 2014;23: 539-551.

12. Kaphingst KA, Ivanovich J, Biesecker BB, et al. Preferences for return of incidental findings from genome sequencing among women diagnosed with breast cancer at a young age. Clin Genet. 2016;89:378-384.

13. Yushak ML, Han G, Bouberhan S, et al. Patient preferences regarding incidental genomic findings discovered during tumor profiling. Cancer. 2016:122:1588-1597.

14. Sanderson SC, Linderman MD, Suckiel SA, et al. Motivations, concerns and preferences of personal genome sequencing research participants: baseline findings from the HealthSeq project. Eur J Hum Genet. 2016; 24:153.

15. Ferrer RA, Taber JM, Klein WMP, Harris PR, Lewis KL, Biesecker LG. The role of current affect, anticipated affect and spontaneous self-affirmation in decisions to receive self-threatening genetic risk information. Cogn Emot. 2014;29:1456-1465.

16. Haga SB, O’Daniel JM, Tindall GM, Lipkus IR, Agans R. Public attitudes toward ancillary information revealed by pharmacogenetic testing under limited information conditions. Genet Med. 2011;13:723-728.

17. Biesecker LG, Mullikin JC, Facio FM, et al. The ClinSeq project: piloting large-scale genome sequencing for research in genomic medicine. Genome Res. 2009;19:1665-1674.

18. Taber JM, Klein WMP, Ferrer RA, Lewis KL, Biesecker LG, Biesecker BB. Dispositional optimism and perceived risk interact to predict intentions to learn genome sequencing results. Health Psychol. 2015;34:718-728.

19. Taber JM, Klein WMP, Ferrer RA, et al. Perceived ambiguity as a barrier to intentions to learn genome sequencing results. J Behav Med. 2015;38: 715-726.

20. Foreman AK, Lee K, Evans JP. The NCGENES project: exploring the new world of genome sequencing. N C Med J. 2013;74:500-504.

21. Sanderson SC, O'Neill SC, Bastian LA, Bepler G, McBride CM. What can interest tell us about uptake of genetic testing? Intention and behavior amongst smokers related to patients with lung cancer. Public Health Genomics. 2010;13:116-124.

22. Sheeran $P$, Maki A, Montanaro $E$, et al. The impact of changing attitudes, norms, and self-efficacy on health-related intentions and behavior: a meta-analysis. Health Psychol. 2016;35:1178-1188.
23. Sandberg T, Conner M. Anticipated regret as an additional predictor in the theory of planned behaviour: a meta-analysis. $\mathrm{Br} J \mathrm{Soc}$ Psychol. 2008;47(Pt 4):589-606.

24. Berg JS, Foreman AK, O'Daniel JM, et al. A semiquantitative metric for evaluating clinical actionability of incidental or secondary findings from genome-scale sequencing. Genet Med. 2016;18:467-475.

25. Karnofsky DA, Burchenal JH. The clinical evaluation of chemotherapeutic agents in cancer. In: MacLeod CM (ed). Evaluation of Chemotherapeutic Agents. Columbia University Press: New York, 1949:191-205.

26. Wingard JR, Curbow B, Baker F, Piantadosi S. Health, functional status, and employment of adult survivors of bone marrow transplantation. Ann Intern Med. 1991;114:113-118.

27. Davis TC, Crouch MA, Long SW, et al. Rapid assessment of literacy levels of adult primary care patients. Fam Med. 1991;23:433-435.

28. Davis TC, Long SW, Jackson RH, et al. Rapid estimate of adult literacy in medicine: a shortened screening instrument. Fam Med. 1993;25: 391-395.

29. Schwartz LM, Woloshin S, Black WC, Welch HG. The role of numeracy in understanding the benefit of screening mammography. Ann Intern Med. 1997; 127:966-972.

30. Ziarnowski KL, Brewer NT, Weber B. Present choices, future outcomes: anticipated regret and HPV vaccination. Prev Med. 2009;48:411-414.

31. U.S. Census Bureau. 2010-2014 American Community Survey 5-year estimates: North Carolina. https://factfinder.census.gov/faces/tableservices/ jsf/pages/productview.xhtml?src= bkmk. Accessed 3 November 2016.

32. Brewer NT, DeFrank JT, Gilkey MB. Anticipated regret and health behavior: a meta-analysis. Health Psychol. 2016;35:1264-1275.

33. Facio FM, Brooks S, Loewenstein J, Green S, Biesecker LG, Biesecker BB. Motivators for participation in a whole-genome sequencing study: implications for translational genomics research. Eur J Hum Genet. 2011;19:1213-1217.

34. Bollinger JM, Scott J, Dvoskin R, Kaufman D. Public preferences regarding the return of individual genetic research results: findings from a qualitative focus group study. Genet Med. 2012;14:451-457.

35. Bernhardt BA, Roche MI, Perry DL, Scollon SR, Tomlinson AN, Skinner D. Experiences with obtaining informed consent for genomic sequencing. Am J Med Genet A. 2015;167A:2635-2646.

36. Tomlinson AN, Skinner D, Perry DL, Scollon SR, Roche MI, Bernhardt BA. "Not tied up neatly with a bow": professionals' challenging cases in informed consent for genomic sequencing. J Genet Couns. 2016;25:62-72.

37. van Harreveld F, van der Pligt J, de Liver YN. The agony of ambivalence and ways to resolve it: introducing the MAID model. Pers Soc Psychol Rev. 2009;13:45-61.

38. Webb TL, Sheeran P. Does changing behavioral intentions engender behavior change? A meta-analysis of the experimental evidence. Psychol Bull. 2006;132:249-268.

39. Abraham C, Sheeran P. Acting on intentions: the role of anticipated regret. Br J Soc Psychol. 2003;42:495-511.

40. Doyle DL, Awwad RI, Austin JC, et al. 2013 review and update of the genetic counseling practice based competencies by a Task Force of the Accreditation Council for Genetic Counseling. J Genet Couns. 2016;25: 868-879. 